\title{
A Case Study on the Proper Use of Human Tissues for Biomedical Research at an Academic Pathology Institution in Switzerland
}

\author{
Stephan C. Schäfer Hans-Anton Lehr \\ Institut Universitaire de Pathologie, Centre Hospitalier Universitaire Vaudoise, Lausanne, Switzerland
}

\author{
Key Words \\ Biobank · Pathology $\cdot$ Regulations $\cdot$ Human tissue • \\ Ethical review board
}

\begin{abstract}
Any academic pathologist will sooner or later be confronted with the need to use human tissues for quality control purposes or for research projects or else with the demand for human tissue samples for research projects of external researchers. Over the last 10 years, the use of human tissues for such non-diagnostic purposes has been the subject of extensive debate and wide-reaching regulations. In particular, questions of medical secrecy, safety, autonomy and anonymization have been addressed, and the role of ethical review boards defined. However, these guidelines are not uniform in all countries and unfortunately tend to suffer from a certain lack of precision, which may in part be due to the fact that they are usually edited by multiple authors with quite diverse backgrounds (physicians, lawyers, ethicists, philosophers, anthropologists, theologists or lay persons, for example). The wide spectrum of interpretations of such regulations may be embarrassing to such an extent that a continuation of academic activities with human tissues appears to many pathologists as almost impossible or even outright illegal. This paper describes a set of characteristic, recurrent situations to which an academic pathologist may be confronted and proposes simple, realistic solutions that are nevertheless in line with most current regulations.
\end{abstract}

Copyright $\odot 2007$ S. Karger AG, Basel
(C) 2007 S. Karger AG, Basel

$1015-2008 / 07 / 0744-0259 \$ 23.50 / 0$

Fax +4161306 1234

E-Mail karger@karger.ch

www.karger.com
Accessible online at:

www.karger.com/pat

\section{Introduction}

In our daily diagnostic activities, our reports on human tumors extend well beyond the pure assignment of a diagnostic entity to the assessment of prognostically relevant parameters, such as tumor cell differentiation, vascular invasion, expression of hormone receptors, to name only a few. In doing so, we pathologists are well aware of the fact that important prognostic information is to be obtained from the study of human tissue samples. This information concerns not only existing lesions, but may also extend to the potential development of future disease. This is ever more burning since the application of modern molecular techniques to minute, fresh or formaldehyde-fixed tissue fragments allows to predict genetic predisposition to disease. Hence, we pathologists assume the responsibility to properly safeguard the human tissue samples that we have been entrusted from improper and unauthorized $(\mathrm{ab})$ use.

Pathology institutes have to keep paraffin blocks for many years (up to 50 years in the case of pediatric tumors). According to an enlarged definition, these archives represent 'biobanks'. The tissue collections in the caves of our pathology institutes are primarily meant to serve the affected patients. For instance, a comparison of a newly developed tumor with preexisting tumors will define whether the new tumor represents an independent new primary or else a prognostically ominous recurrence. Also, investigations into the expression of antigens on archived tumor samples will help us to define the po-

Prof Hans-Anton Leh

Institut Universitaire de Pathologie

Centre Hospitalier Universitaire Vaudoise, Rue du Bugnon 25

$\mathrm{CH}-1011$ Lausanne (Switzerland)

Tel. +41 21314 7120, Fax +41 21314 7205, E-Mail hans-anton.lehr@chuv.ch 
tential benefit of novel targeted therapies that may not have been available when the tumor was first diagnosed (e.g. Her2/neu or CD117).

However, this stored tissue can also be used for biomedical research. In the light of the initially described dangers of an unauthorized or careless use of human tissue on the one hand and the benefits of making human tissue available for biomedical research on the other hand, we pathologists welcome the worldwide initiatives for a better regulation of the correct use of human tissues. It is the aim of this paper to describe - using a series of typical research projects - how these guidelines can be applied in an academic pathology setting.

Pathology institutes are very special places. Pathologists officially receive human tissues along with request forms that contain more or less detailed clinical information, e.g. specific clinical manifestations, previous diagnoses, results of non-invasive imaging or therapy response. This clinical information is meant to help us in our quest for an effective, optimal diagnosis of the underlying pathology. As such, we pathologists are included in the patient's physician team and are bound by the medical secret. This also means that we have to safeguard the tissues and assure that no abuse is possible.

\section{Regulations}

In Switzerland, the Swiss Academy for Medical Sciences (SAMW) has issued guidelines for the proper use of human tissues for research and teaching purposes. These guidelines have been discussed and the final version modified according to external input by scientists and clinicians (www.SAMW.ch). The final version has been officially commented by the directors of the five Swiss university pathology institutes and the interpretations largely consented to by the SAMW (www.swissethics.ch/ Ethische Normen und Prinzipien/Korrespondenz zwischen den universitären Pathologie-Instituten und der SAMW). While these guidelines have no binding legal character, they do represent the present code of conduct and will eventually be followed by a national law on research in human subjects that is scheduled to see the light of day not before the year 2010. These regulations are most appropriate and largely recognize the need for scientific progress. Nevertheless, the efforts to reconcile the most divergent viewpoints of physicians, scientists, lawyers, ethicists, theologists and lay people have often resulted in confusing and sometimes contradictory formulations (see correspondence with SAMW stated above).
So, how is it still possible that human tissue can be rightly used for biomedical research? First, we should not forget that during tumor surgery not only the tumor itself is removed, but also a safety rim of non-neoplastic tissue, and that during macroscopic examination of the resection specimen, only a few representative tissue blocks are selected for later histological examination. The rest of the specimen is considered 'surplus' tissue that will eventually be discarded. This surplus tissue constitutes an ideal source of tumoral and non-tumoral tissue samples that can be used for biomedical research without jeopardizing the accuracy of the histopathological examination. The more important question now pertains to how we will use these tissue samples in an ethically and legally correct manner in order to prevent any potential abuse.

In the following, we will describe a series of typical scenarios that we encounter during our academic activities as diagnostic pathologists. Note that the situations and solutions proposed herein are based on the personal experience of the two authors in the legal and ethical frameworks of their present and past professional environment (Centre Hospitalier Universitaire Vaudois, University of Lausanne, Switzerland, and formerly the University Hospital of the Johannes Gutenberg University of Mainz, Germany).

\section{Typical Scenarios}

\section{Case 1}

A scientist in a physiological institute of our medical school found a new receptor variant for a well-established tumor-associated growth factor. She characterized this receptor variant using refined molecular techniques, transferred the receptor to a mouse model and thus found a key role of this receptor variant on the growth of experimental colorectal carcinomas. In the colonic tumors of her mice, the receptor was overexpressed and its targeted inhibition led to apoptosis and involution of the tumors. Is this exciting finding relevant for patients with colon cancer? If she does not herself ask this question, then the reviewers of her manuscripts in a prestigious scientific journal will for sure.

This question can be addressed by first examining immunohistochemically the expression of this receptor variant in a small series of human colorectal carcinomas. For that purpose, the researcher requires human tumors and she will likely ask for such samples in the neighboring pathology institute. Any academic pathologist will 
have been confronted with such requests and will likely have responded positively. For the purpose of such a tissue transfer, patient identifiers are carefully removed from the tumor blocks and handed over to the researcher either completely anonymized (designated 'colon carcinoma 1' for example) or associated with a project-specific code which will remain in the possession of the pathologist or of a specifically assigned trustee. Pathology institutes should require a brief documentation of the scientific rationale, some details concerning project logistics (e.g. who will prepare cryosections and who will pick them up), as well as the names and affiliations of all persons involved in the study. Also, the project leader should sign a statement that he will discard the samples after having used them for his experiments and will not keep them for future research projects. This information should typically be documented on a standardized form which will be kept in a safe place and regularly or upon specific request brought to the attention of the local ethical review board. Before handling of the specimen, the pathologist has the duty to verify that only such tissues are relinquished which will not, under any circumstances, be required for potential investigations for the benefit of the tissue donor. For that purpose, one or two blocks of the tumor will be kept in the archives. Also, academic pathology institutes nowadays tend to keep cryopreserved tissue for later molecular pathologic investigations for the benefit of the affected patient.

\section{Ethical-Legal Considerations}

For the use of anonymized or linked anonymized tissue samples, a general consent to biomedical research should suffice. Such consent might be obtained during the admission of the patient to the hospital or else in parallel to the consent to the surgical intervention. We recommend maximum transparency with regard to any research projects on human tissues and advise pathologists to negotiate clear and transparent rules with their local ethical review boards concerning the disclosure of ongoing research projects. Some institutions have requested that any research project using human tissue should be submitted and officially approved by an ethical review board. Such propositions, which degrade ethical review boards to a pseudoscientific quality police, may appear exaggerated, considering the virtual non-existence of potential dangers originating in the transfer of anonymized or linked-anonymized tissue samples. Alternative solutions appear more suitable, such as the standardized disclosure of any new research project using human tissue to members of the review board which may then choose selected projects for an in-depth review. Also, a simplified, accelerated review process for all research projects on anonymized or linked-anonymized tissue samples might be a reasonable solution.

\section{Case 2}

As part of a multicenter therapeutic study, tumor tissue is requested to be sent to an external laboratory for immunohistochemical and molecular pathologic investigations. The identity of the tissue donor will be replaced by a project-specific working code on the tissue block.

\section{Ethical/Legal Considerations}

For such a situation, the transfer of tissue by the pathologist requires a project-specific informed consent by the patient. The project-specific working code on the tissue block does not qualify as a code in the sense of linked anonymization since tissue is transferred to a third person outside of the pathology institute (typically a pharmaceutical company) who will be able to establish a link between the tissue and the patient's identity. Such tissue transfer can under no circumstances be based on a general or even presumed consent. In our daily routine, we do not verify the signature of the patient but will document in our files the provision of such informed consent in the study protocol as well as the acceptance letter by the ethical review board. Since pathologists are usually involved in the final version of the study protocol, we do verify that the planned transfer of tissue to an external third person is explicitly mentioned in the protocol as well as in the informed consent form. It stands to reason to specify whether the use of the transferred tissue is limited to questions related to the specific research protocol or whether tissue may subsequently be used by the external third person for secondary research protocols. Such an enlarged use of tissues obtained during specific therapy trials by pharmaceutical companies is common practice and we believe that the concerned patient should be informed of this prospect before he gives his consent to the protocol.

\section{Case 3}

During his many night calls, a radiologist has quantified by image analysis the size reduction of primary breast carcinomas under three different neoadjuvant therapy protocols. He now asks his pathologist for cancer tissue samples of these patients in order to study whether genetic printing can help predict therapy response of one or 
the other neoadjuvant chemotherapy protocols for future patients.

\section{Ethical/Legal Considerations}

Version $3 A$. If the genetic fingerprinting and/or the chemotherapy response data require association to patientspecific parameters (i.e. body mass index, lifestyle factors, compliance, dose reduction or complications), then the study cannot be performed in an anonymized or even a linked-anonymized form. Even though the potential harm for the patient is exceedingly small, the fact that the study protocol previews the examination of the tissue in a personalized form means that the patients have to sign a projectspecific informed consent. It goes without say that an ethical review board will first have to approve the protocol.

Version $3 B$. If, however, intelligent stratification algorithms can be formulated that allow to place patients in various subgroups (i.e. patients who have received therapy $\mathrm{C}$ and whose tumors have shown a size reduction $>50 \%$ ), then a linked anonymized transfer of tissue samples is possible, which could then be covered by a general consent. We typically require that the subgroups contain a minimum of 5 patients so that identification of the patients within the subgroups is not easily possible. As delineated above, the protocol of any such study is documented and brought to the attention of the ethics review board which may then decide whether or not they want to officially review and approve the study.

\section{Case 4}

A pathologist wants to use a tissue block of a Her2/ neu-overexpressing breast carcinoma of one of his patients to control for effective immunostaining of breast carcinomas in subsequent patients.

\section{Ethical/Legal Considerations}

Such a use of human tissue for quality control falls into the realm of primary diagnosis and as such is not considered biomedical research. This use is even part of good clinical practice and as such does not necessitate patient consent or approval by the ethical review board.

\section{Case 5}

A pathologist wants to investigate whether a new antibody which has been reported to show high specificity for urothelial carcinomas does live up to these expecta- tions and could hence be used in daily diagnostic use. Based on past disappointments, where the reported specificity tends to diminish with the number of publications, the pathologist reasons that internal verification of specificity and sensitivity of the antibody is necessary before the antibody can be applied in routine diagnostic tests.

Such an investigation of specificity and sensitivity is typically addressed by comparing a significant number $(n=25)$ of well-characterized urothelial carcinomas with an equally large number of carcinomas at other sites (i.e. $\mathrm{n}=10$ breast cancers, 10 colon cancers and 10 thyroid cancers) in terms of specific expression of this new marker. For such studies, we nowadays tend to use multi-tissue blocks which guarantee standardized conditions during immunohistochemical incubations. The pathologist will use his archive for the generation of such tissue blocks and may document tumor-specific parameters (i.e. welldifferentiated, intermediately differentiated, fully differentiated urothelial carcinomas). He will work in-house with patient-specific pathology numbers in order to allow for follow-up on unexpected results (which might be encountered when the marker is expressed in a control prostate cancer, which upon review turns out to be a collision tumor between a prostate and an urothelial carcinoma).

\section{Ethical/Legal Considerations}

Tissues and diagnoses remain during the entire study in the hands of the pathologist, who is bound by the medical secret concerning his patients. The possibility of patient identification along patient-specific pathology codes solely serves quality control purposes. Since no tissue is transferred out of the realms of the pathology institute and since no adverse events can be construed for the tissue donor, a specific consent by the patient is not required. The project has to be documented just as any other research project on the standardized form and brought to the attention of the ethical review board. In case that the ethical review board may request to review the research project, this request will of course be respected and the project started only upon approval by the ethical review board.

\section{Case 6}

A nother typical pathology project: a pathologist wants to know whether cervical condylomas with marked dysplasia tend to progress faster towards invasive carcinoma 
if a particularly aggressive subtype of human papilloma virus can be documented. For this study, several procedural scenarios are possible.

Scenario $6 \mathrm{~A}$. The pathologist will investigate a continuous series of 100 cervical carcinomas with marked dysplasia by in situ hybridization (which, of course, will not be billed to the patient) and asks his clinical colleague to inform him about the subsequent clinical course of the disease, as well as on potential repeat biopsies in other clinics. A project-specific follow-up procedure and re-biopsies are not part of his protocol.

Scenario $6 B$. Same as above, but this time with regularly scheduled annual follow-up visits involving biopsy controls of all patients entered into the study.

Scenario 6C. The pathologist selects 100 continuous cervical condylomas with marked dysplasia and sends paraffin blocks of these lesions to an external research laboratory for more sophisticated subtyping of the human papilloma virus. For this purpose, any patient identifiers will be removed from the tissue blocks and replaced by a project-specific code to which he holds on to. Upon return, the outside test results will be linked by the pathologist to the patients in order to allow association of diagnosis of human papilloma virus with the clinical course. For that purpose, he asks his clinical colleague to inform him about the subsequent clinical course of the disease, as well as on potential re-biopsies in other clinics. A project-specific follow-up procedure and re-biopsis are not part of his protocol.

\section{Ethical/Legal Considerations}

Scenarios $6 \mathrm{~A}$ and $6 \mathrm{C}$. A specific consent is not indicated since the tissue will remain in the hand of the pathologist (6A) or is being transferred in a linked-anonymized way into the hands of an external research laboratory (6C); the study can be performed based on a general consent. The project must be documented on a standard form and brought to the attention of the ethical review board for potential in-depth review.

Scenario $6 B$. The protocol requires project-specific follow-up and re-biopsies of all patients, which requires review of the protocol by the ethical committee as well as a project-specific informed consent by the patient.

\section{Concluding Remarks}

The proposed solutions for the above-described scenarios can be realized in daily routine without any problem. They respect the safety and autonomy of our patients. They respect the role of the ethical review boards as important control organs. At the same time, the strictly applied and well-standardized mode of anonymization of tissue serves to prevent any harm to the patients and helps to avoid that each and every unproblematic protocol must be individually reviewed and approved by the review board. Positive aspects for the researchers include the direct and rapid response to their needs for human tissues. Finally, members of pathology institutions may respond to their academic role as valuable collaborators in biomedical research through the well-organized and well-controlled retrieval, storage and transfer of human tissue. 